MARCOS VINÍCIUS MELCONIAN

\title{
MODELAGEM NUMÉRICA E COMPUTACIONAL COM SIMILITUDE E ELEMENTOS FINITOS
}

Desenvolvimento de Equação Preditiva para o Cálculo da Força de Retenção em Freios de Estampagem

SÃO PAULO

2014 


\section{Modelagem Numérica e Computacional com Similitude e Elementos Finitos:}

Equação preditiva geral para o cálculo da força de retenção em freios de estampagem

(C) 2014

1 st edition - 2014

Editora Edgard Blücher Ltda.

ISBN: 978-85-8039-090-2

\section{Blucher}

Rua Pedroso Alvarenga, $1245,4^{\circ}$ andar

04531-012 - São Paulo - SP - Brazil

Fax 551130792707

Phone 551130785366

editora@blucher.com.br

www.blucher.com.br

Segundo o Novo Acordo Ortográfico, conforme 5a ed. do Vocabulário Ortográfico da Língua Portuguesa, Academia Brasileira de Letras, março de 2009.

É proibida a reprodução total ou parcial por quaisquer meios, sem autorização escrita da Editora.

Todos os direitos reservados pela

Editora Edgard Blucher Ltda.

\section{FICHA CATALOGRÁFICA}

Melconian, Marcos Vinícius

Modelagem numérica e computacional com similitude e elementos finitos : equação preditiva geral para o cálculo da força de retenção em freios de estampagem [livro eletrônico] / Marcos Vinícius Melconian. -- São Paulo : Blucher, 2014.

$3 \mathrm{Mb}$; ePUB.

Bibliografia

ISBN 978-85-8039-090-2 (e-book)

ISBN 978-85-8039-089-6 (impresso)

1. Engenharia Mecânica - ecnologia 2. Método dos elementos finitos 3. Estampagem (Metais) I. Título

$14-0612$

CDU 623.045

Índices para catálogo sistemático:

1. Engenharia Mecâni 


\section{Dedicatória}

Dedico este trabalho a Deus, aos meus pais Sarkis e Anaid, e ao meu irmão Sérgio, por serem à base da minha vida e inspiração.

\section{AGRADECIMENTOS}

A realização e conclusão deste trabalho passaram pela ajuda, apoio e dedicação de muitas pessoas. Gostaria de agradecer principalmente:

Ao Luis Fernando que me ajudou com os primeiros passos, na utilização do STAMPACK ${ }^{\circledR}$.

Agradeço aos pesquisadores da QUANTECH ATZ, em especial Albert Forgas, por toda ajuda e disponibilidade com a utilização do STAMPACK ${ }^{\circledR}$. Aos professores, funcionários e colegas de classe do Instituto Federal de Educação, Ciência e Tecnologia de São Paulo e de Bragança Paulista por toda a ajuda, dedicação, apoio e amizade.

Por fim, e de maneira muito especial, agradeço ao meu orientador Prof. Dr. Écio Naves Duarte. Obrigado por todas as inúmeras aulas e aconselhamentos. Sempre indicando os melhores caminhos para o desenvolvimento e conclusão deste trabalho, com muita disposição e compromisso e aos meus familiares por toda ajuda e incentivo.

"Predição é muito difícil, especialmente se for sobre o futuro." 


\section{SUMÁRIO}

\section{INTRODUÇÃO................................................ 11}

1.1 Objetivos e justificativas do trabalho.................................15

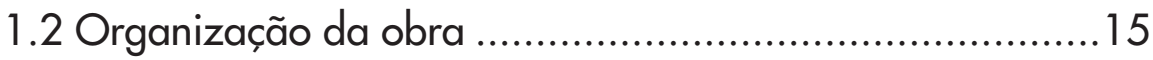

2. REVISÃO BIBLIOGRÁFICA............................... 17

3. FUNDAMENTAC̣ÃO TEÓRICA .............................. 25

3.1 Estampagem de chapas metálicas ..................................26

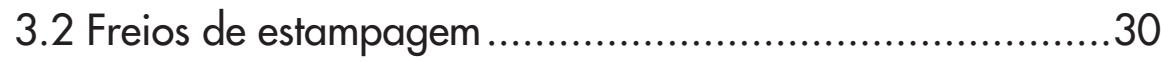

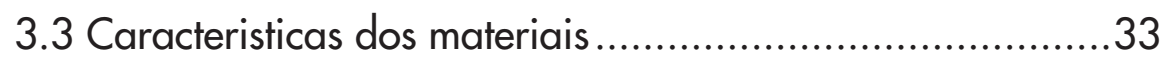

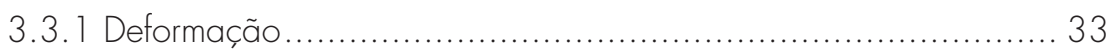

3.3.2 Deformação elástica............................................................. 33

3.3.3 Deformação plástica............................................................. 34

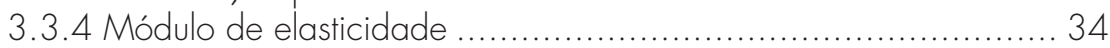

3.3.5 Limite convencional de elasticidade .......................................36 36

3.3.6 Lei de encruamento isotrópico ……..................................... 37

3.3.7 Anisotropia .................................................................. 39

3.3.8 Critério de plasticidade ........................................................ 41

3.4 Similitude em engenharia............................................44

3.4.1 Descrição da metodologia................................................ 44

3.4.2 Obtenção das equações preditivas ...................................... 47

3.50 método dos elementos finitos .......................................49

3.5.1 Descrição geral do método.............................................. 49

3.5.2 Passos para a análise e solução em elementos finitos ................ 52

3.5.2.1 $\bigcirc$ processo de discretização do método ............................ 53

3.5.2.2 Definição das propriedades dos elementos ......................... 54

3.5.2.3 Montagem das matrizes de rigidez dos elementos ................ 55

3.5.2.4 Aplicar as cargas.................................................. 56

3.5.2.5 Definir as condições de contorno ................................... 56

3.5.2.6 Solucionar o sistema de equações .................................. 57

3.5.2.6 Calcular os esforços .................................................. 57

3.5.3 Fases do método dos elementos finitos .............................. 57

3.5.3. 1 Pré-processamento .................................................... 57

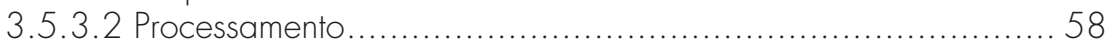

3.3.3.3 Pós-processamento ................................................... 58 
3.6 Solução implícita versus solução explícita

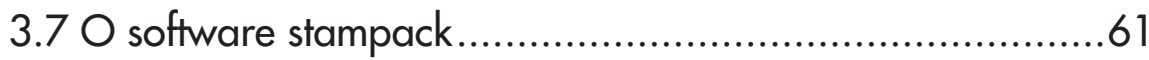

\section{METODOLOGIA ............................................... 65}

4.1 Bases de dados experimentais.........................................66

4.1.1 Experimento realizado ...................................................... 66

4.1.2 A modelagem do freio de estampagem ............................. 72

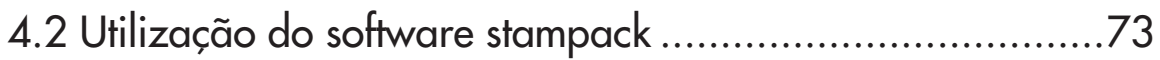

4.2. 1 Importação da figura ...................................................... 74

4.2.2 Configuração das variáveis .......................................... 74

4.2.3 Resultados da simulaçâo ................................................ 78

\section{DESENVOLVIMENTO DA EQUAC̣ÃO}

PREDITIVA GERAL (EPG)...................................... 79

5.1 Validações do modelo em elementos finitos ........................80

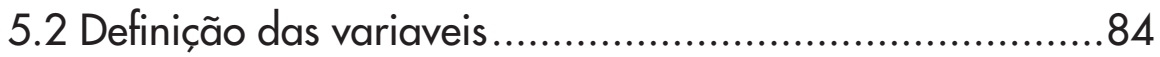

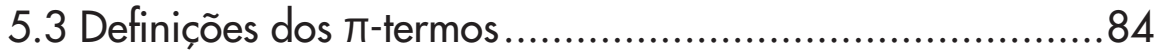

5.4 Obtenção das equações componentes ...............................86

6. RESULTADOS OBTIDOS....................................... 103

6.1 Validação das equações com os dados experimentais.....104

6.2 Desenvolvimento do aplicativo para cálculo ...................106

6.2.1 Escolha da plataforma e linguagem de programação ............. 107

6.2.2 Apresentação do aplicativo .......................................... 107

\section{CONCLUSÕES .............................................. 109}

7.1 Conclusões sobre a pesquisa........................................110

7.2 Sugestões para trabalhos futuros ..................................113 


\section{LISTA DE FIGURAS}

Figura 1 - Representação do freio de estampagem no processo, (CADELL R.M, HOSFORD W.F, 2000) ................12

Figura 2 - Imagem da deformaç̦ão da chapa em função da penetração do freio, (KEELER, 2000) .......................20

Figura 3 - Chapa se movendo em um freio retangular, (KEELER, 2000) ....................................................21

Figura 4 - llustração do freio de estampagem, (ZHHHENG, 2011) ...............................................................22

Figura 5 - Testes com os resultados experimentais de Nine (1978)

e com os analíticos de Stoughton (1988), disponíveis em Guo et al. (2000) ..............................23

Figura 6 - Peça tracionada com detalhe da estricção do material ..................................................................26

Figura 7 - (A) Prensa Hidráulica com carga nominal máxima de 5000kN;

(B) Prensa mecânica com carga nominal máxima de 35000kN [Schuler, Inc.] ............................27

Figura 8 - Prensa mecânica de acçoo simples [Schuler, Inc.] .........................................................................27

Figura 9 - Prensa mecânica de dupla ação [Schuler, Inc.] ........................................................................28

Figura 10 - (A) Rugas no flange (B) Rugas laterais, (REDDY, 2012) .......................................................29

Figura 11 - (A) Freio de estampagem e a matriz antes do contato. (B) Freio de estampagem

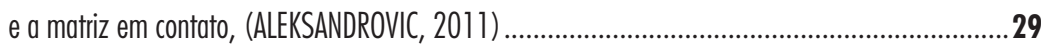

Figura 12 - Diagrama de flexão e flexão inversa em um freio de estampagem..............................................30

Figura 13 - Gráfico Tensão x Deformaç̦ão, (MELCONIAN, 2008)...............................................................35

Figura 14 - Diagrama tensão deformação, para determinaç̦̃o da tensão limite convencional de elasticidade $(\sigma e) \mathbf{3 6}$

Figura 15 - Determinaç̃ão de K e n..................................................................................................38

Figura 16 - Curvas das leis de encruamento de Ludwik- Nadai e Voce em um gráfico de Tensão x Deformaç̃õo para uma aço - FeP03_A, (STAMPACK $\left.{ }^{\circledR}\right)$..........................................38

Figura 17 - Corpos de prova para realização de testes para determinar a anisotropia do material .........................40

Figura 18 - Representação das tensões hidrostáticas em um corpo ...............................................................42

Figura 19 - Representação gráfica do efeito Bauschinger ....................................................................43

Figura 20 - Malha triangular plana, (SOUZA, 2003) ..............................................................................5

Figura 21 - Diferentes tipos de elementos finitos, (SOUZA, 2003) ...........................................................51

Figura 22 - Graus de liberdade. A) graus de liberdade de um ponto; B) graus de liberdade de um corpo rígido ....52

Figura 23 - Passos para solução em Elementos Finitos .......................................................................53

Figura 24 - Discretização de diferentes sólidos e estruturas com o MEF, (ONÃTE, 2009) .................................54

Figura 25 - Exemplos de peças discretizadas ......................................................................................5

Figura 26 - Relação de aspecto de elementos retangulares ................................................................55

Figura 27 - Força externa aplicada na mola .......................................................................................56

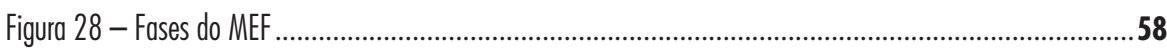

Figura 29 - Aplicações do método explíito e método implíito ............................................................60

Figura 30 - Vis̃̃o global da simulaç̃õo em EF, (STAMPACK $\left.{ }^{\circledR}\right)$.....................................................................63

Figura 31 - Prensa-chapas segurando a chapa metálica que será estampada, (NINE, 1978) ..............................66

Figura 32 - Aparato com freios rolantes, (NINE, 1978) ..........................................................................66

Figura 33 - Esquema de montagem dos freios rolantes, (NINE, 1978) ................................................67 
Figura 34 - Esquema de montagem dos freios rolantes, (NINE, 1978) ......................................................67

Figura 35 - Aparato utilizado na montagem dos freios de estampagem, (NINE, 1978) ..................................69

Figura 36 - Freio de estampagem desenhado no software SolidEdge ST5 ...............................................71

Figura 37 - Aplicaçōes do STAMPACK ${ }^{\circledR}$......................................................................................72

Figura 38 - Imagem do freio de estampagem importada para o software STAMPACK

Figura 39 - Malha não estruturada discretizada por erro cordal .................................................................74

Figura 40 - Malha estruturada retangular ...............................................................................................74

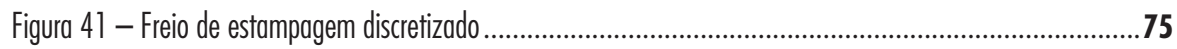

Figura 42 - Valores das propriedades mecânicas da chapa metálica, adotados em uma simulação típica (STAMPACK $\left.{ }^{\circledR}\right)$....................................................................76

Figura 43 - Pró-processamento do freio de estampagem......................................................................77

Figura 44 - Geometria dos freios de estampagem..............................................................................80

Figura 45 - Gráfico da Força de retenção do freio (N) x Tempo(s), em uma simulação feita no STAMPACK ${ }^{\circledR}$.......82

Figura 46 - Gráfico com resultado das simulações para $\pi 1 \times \pi 2$.............................................................92

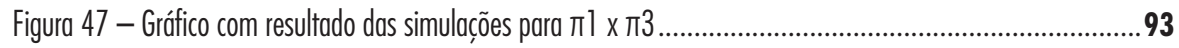

Figura 48 - Gráfico com resultado das simulações para $\pi 1 \times \pi 4$............................................................94

Figura 49 - Gráfico com resultado das simulaçōes para $\pi 1 \times \pi 5$............................................................95

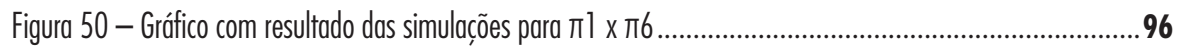

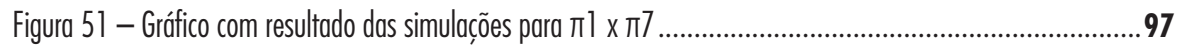

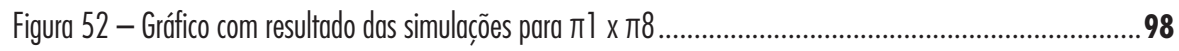

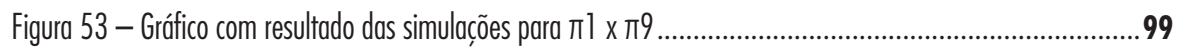

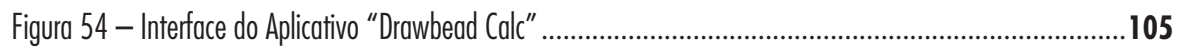

Figura 55 - Análise de sensibilidade da FR em relação a cada parâmetro persente na EPG elaborada..................108

Figura 56 - Análise de sensibilidade da FR em relação a cada parâmetro persente na EPG elaborada..................108 


\section{LISTA DE TABELAS}

Tabela 1 - Modelos propostos para a FR e FP, de acordo com Xu et al. (1998), apud Duarte (2007)

Tabela 2 - Geometrias das seçōes de freios mais usuais. a) Semicircular; b) Retangular; c) Triangular d) Trapezoidal; e) Assimétrica - XU et al., 1998 apud DUARTE, 2007 (adaptado) .............................31

Tabela 3 - Propriedades mecânicas dos materiais utilizados nos experimentos, (NINE, 1978) ...........................68

Tabela 4 - Forças de retenção dos freios para chapas de aço, (NINE, 1978)............................................70

Tabela 5 - Forças de retenção dos freios para chapas de aluminio, (NINE, 1978)...........................................70

Tabela 6 - Parâmetros utilizados experimentalmente, (NINE 1978) ......................................................81

Tabela 7 - Resultados obtidos para validação do modelo utilizado nas simulações com o STAMPACK ${ }^{\circledR}$.................82

Tabela 8 - Natureza de cada parâmetro .....................................................................................................83

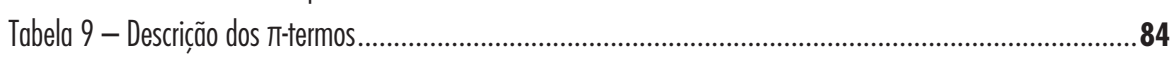

Tabela 10 - Valores adotados para cada parâmetro...........................................................................86

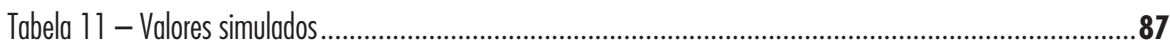

Tabela 12 - Obtenção dos pontos para os $\pi$-termos .....................................................................89

Tabela 13 - Intervalo de validade de cada parâmetro ............................................................................91

Tabela 14 - Validação das EPGs obtidas, com os dados experimentais ...................................................101

Tabela 15 - Parâmetros utilizados para 0 teste da EPG ...............................................................102

Tabela 16 - Comparativo dos resultados obtidos or meio da simulação em EF e calculados pela EPG .................103

Tabela 17 - Comparativo dos limites de validade dos $\pi$ - termos

da EPG de Duarte (2007) e de Melconian (2014) ..................................................................110 


\section{LISTA DE ABREVIATURAS}

$$
\text { ABREVIATURAS }
$$

\begin{tabular}{|c|l|}
\hline EF & Elementos Finitos \\
\hline EPG & Equação Preditiva Geral \\
\hline FP & Força do Prensa- Chapas \\
\hline FR & Força de Retenção do Freio \\
\hline MEF & Método dos Elementos Finitos \\
\hline
\end{tabular}




\section{LISTA DE SÍMBOLOS}

SÍMBOLOS

\begin{tabular}{|c|c|}
\hline$E$ & Modulo de Young \\
\hline$\varepsilon$ & Deformação \\
\hline$F$ & Força Aplicada \\
\hline$k$ & Constante de proporcionalidade do material \\
\hline$\Delta x$ & Variação de comprimento \\
\hline$K$ & Modulo de encruamento \\
\hline$n$ & Expoente de encruamento \\
\hline$\sigma$ & Tensão mecânica \\
\hline$\sigma_{e}$ & Tensão limite convencional de elasticidade \\
\hline$\sigma^{y}$ & Tensão de escoamento final \\
\hline$\sigma^{e}$ & Tensão de escoamento inicial do material \\
\hline$\Delta r$ & Anisotropia planar \\
\hline$r$ & Anisotropia transversal \\
\hline$\sigma_{m}$ & Tensão Hidrostatica \\
\hline$\sigma_{p}$ & Tensão de proporcionalidade \\
\hline$\pi_{i}$ & Pi-termo \\
\hline$r_{o}, r_{45}$ e $r_{90}$ & Coeficiente de Lankford \\
\hline$\{F\}$ & Matriz coluna com todas as cargas nodais \\
\hline$\{U\}$ & Matriz de rigidez da estrutura \\
\hline$[K]$ & Matriz coluna com todos os deslocamentos nodais \\
\hline$v$ & Velocidade \\
\hline$v_{s}$ & Velocidade de propagação do some $m$ um material \\
\hline$v$ & Coeficiente de Poisson \\
\hline$\rho$ & Massa especifica \\
\hline$R_{m}$ & Raio da matriz \\
\hline$R_{d}$ & Raio do Freio \\
\hline$c$ & Folga horizontal entre o freio e a chapa \\
\hline$C_{\alpha}$ & Constante em Função dos Grupos de Variáveis Adimensionais \\
\hline$t$ & Espessura da chapa \\
\hline$h$ & Penetração \\
\hline$m$ & Atrito \\
\hline
\end{tabular}

